\title{
AN EMPIRICAL BULK MODULUS MODEL OF TERNARY CHALCOPYRITE STRUCTURE SOLIDS
}

\author{
S. K. Gorai ${ }^{*}$, Guruprasad Parida ${ }^{2}$ and Ajay Kumar Sharma ${ }^{3}$ \\ ${ }^{1}$ Postgraduate Department of Physics, Tata College, Kolhan University, Chaibasa, West \\ Singbhum-833201, Jharkhand, India \\ 2,3 Jharkhand Rai University, Kamre, Ratu Road, Ranchi-835222, Jharkhand, India. \\ *E-mail: shreyagorai@gmail.com
}

\begin{abstract}
A simple empirical relation has been proposed to estimate the bulk modulus (B) of ternary chalcopyrite structure solids of I-II-VI, II-IV-V type from the electronegativies of the constituent atoms and the principal quantum number of atoms of the compounds. The present relation is based on chemical bonding model. The electronegativity and principal quantum number determine the chemical bonding of constituent atoms of compounds. The chemical bonding of compounds is the starting point of the stability of compound which is based on the minimum energy principle. In this study, the ground state property like bulk modulus has been correlated with the nature of bonding of materials. The combination of electronegativity and principal quantum number gives a good account of bonding of ternary chalcopyrite structure solids. These two parameters provide a reliable guide to the structural stability of chalcopyrite structure solids. The computed values for bulk modulus are found to be good agreement with the known ones, the comparison between our present values and the ones in the literature is also given.
\end{abstract}

Keywords: Electronegativity, Bulk modulus, Ternary Chalcopyrite structure, Principal quantum number

(c) RASĀYAN. All rights reserved

\section{INTRODUCTION}

There has been increasing interest in research over three decades of ternary chalcopyrite because of technical importance for linear and nonlinear optical properties. ${ }^{1-3}$ They have wide application in the field of photovoltaic solar cells, light emitting diodes, infrared detectors etc. There are different methods of materials prepared for these chalcopyrite's compounds have been suggested..$^{4-9}$ This structure of chalcopyrite which is obtained from that of zinc blende or sphaerlite by the replacement of cationic sublattice by two different atomic species induces the doubling of the unit cell in a direction, which is conventionally called $\mathrm{c}$ and a tetragonal distortion. The parameter $\eta=c / 2 a$ where $c$ and $a$ as lattice parameter which determines the distortion and by the anion displacement $\mathrm{u}=0.25+\left(\mathrm{d}^{2} \mathrm{~A}-\mathrm{C}+\mathrm{d}^{2} \mathrm{~B}-\mathrm{C}\right) / \mathrm{a}^{2}$ from its position in the cubic cell, where $\mathrm{d}_{\mathrm{A}-\mathrm{C}}$ and $\mathrm{d}_{\mathrm{B}-\mathrm{C}}$ are the cation -anion distances. These ternary chalcopyrite structure solids reveal a wide range of interesting physical and chemical properties. This is due to structural $(\eta, \mathrm{u})$ and chemical ( $\mathrm{d}_{\mathrm{A}-\mathrm{C}}$ and $\mathrm{d}_{\mathrm{B}-}$ ) change of parameters, comparing to binary analog (II-VI and III-V) compounds. The crystals are held in a given geometrical form by virtue of bonding of their atoms with each other. The nature and strength of bonding affect various physical, electrical, magnetic and optical properties of solids. Most solids possess elasticity, which opposes the applied force and tends to restore the body to its original state once the applied force has been removed. The greater the opposition of a solid to deformation, the higher would be its elasticity. Verma and Sharma ${ }^{10-12}$ have recently calculated the electronic, mechanical, and optical properties of semiconductors and insulators with the help of ionic charge theory of solids. No attempt has been made in this direction to correlate bulk modulus with the electronegativity and principal quantum number of valence electrons in atom forming the compounds. In view of the above, a simple empirical relation has been proposed to evaluate the bulk modulus of ternary chalcopyrite's which are in excellent agreement with the previous estimation. ${ }^{13-32} \mathrm{~A}$ fairly good agreement has been obtained between them. 


\section{METHODOLOGY}

The ground state property like bulk modulus is an important physical quantity to account for mechanical strength of the bonds in compounds. It is a property of the material, which shows its resistance to volume changes when compressed. For cubic crystals, it is a critical single material property to show hardness as suggested by theoretically as well as experimentally. Many theoretical approaches have been reported to determine the bulk modulus of solids. ${ }^{19,}{ }^{20}$ The first principle calculation based on density functional theory (DFT) and the DFT plane wave pseudopotential (PW-PP) technique is used for computational studies of structural and quantum mechanical electronic system. ${ }^{21}$ DFT with local density approximation LDA is also used to find out the bulk modulus of metals. Using Murnaghan Equation of state EOS and their P-V relation, using the pressure values, the bulk modulus can be estimated. ${ }^{21}$ The P-V relation of the Murnaghan EOS is:

$$
P=\frac{B}{B_{0}}\left[\left(\frac{V}{V_{0}}\right)^{B_{0}^{I}}-1\right]
$$

From equation (1) we observe that:

$$
d E=-P d V
$$

Using definition the bulk modulus of a solid material is as follows:

$$
B_{0}=-V\left(\frac{\partial P}{\partial V}\right)_{T}=-V\left(\frac{\partial^{2} E}{\partial V^{2}}\right)_{T}
$$

Wang and $\mathrm{Ye}^{19}$ have proposed linear fitting equation of bulk modulus and lattice constants of group IV and III-V zinc blende phase as:

$$
B_{0}=-\alpha+\left(\frac{\beta}{V_{0}}\right)
$$

where, $\alpha$ and $\beta$ have numerical values $-0.45118,0.22137$ for group IV zinc blende phase and -0.32202 , 0.19314 for III-V zinc blende phase. For alkali metals, these values are -0.00367 and 0.00488 respectively. The different values of $\alpha$ and $\beta$ of same geometrical configuration are due to different valence electron character of elements in the different group of the periodic table. For rock salt type crystal structure compounds, Cohen ${ }^{17}$ proposed the relation of isothermal bulk modulus $\mathrm{B}$ in terms of nearest neighbor distance $\mathrm{d}\left(\right.$ in $\mathrm{A}^{\mathrm{o}}$ ) as:

$$
B=550 d^{-3}
$$

For zincblende solids, the following relation was proposed ${ }^{16}$

$$
B=1761 d^{-3.5}
$$

Lam et al ${ }^{18}$ using the local-density formalism and the Pseudopotential approach deduced the relation as:

$$
B=1971 d^{-3.5}-408(\Delta Z)^{2} d^{-4}
$$

where $\Delta Z=1$ and 2 for III $-\mathrm{V}$ and II - VI semiconductors. Verma and Bhardwaj ${ }^{12}$ extended Cohen's relation (6) and (7) to:

$$
B=\left(Z_{1} Z_{2}\right)^{A} N d^{-3}
$$

where $Z_{1}$ and $Z_{2}$ are the ionic charges of the cation and anion respectively, $A$ and $N$ are constants. Their numerical values are 0.75 and 550 for rock salt crystal structure compounds and 0.2 and 750 for zinc blende crystal structure compounds respectively. Liu and Cohen ${ }^{18}$ suggested the following relation for binary crystals: 


$$
B=\frac{\left\langle N_{c}\right\rangle}{4}(1971-221 \lambda) d^{-3.5}
$$

where $N_{c}$ the bulk coordination number,d is the bond length, $\lambda$ is an empirical iconicity parameter takes the value $0,1,2$ for IV,II-VI and III-V semiconductors respectively, the correction factor of the bond ionicity, according to Meng $\mathrm{et} \mathrm{al}^{28}$

$$
\lambda=\frac{\left|G_{A}-G_{B}\right|}{2}
$$

where $G_{A}$ and $G_{B}$ are the numbers of the group of elements A and B respectively in the periodic table. Meng et $a l^{28}$ has used the formula as:

$B^{\mu}=\frac{N_{C}}{4} \frac{1971-221 \lambda}{\left(d^{\mu}\right)^{3.5}}$

wherein $\mathrm{GPa}, d^{\mu}$ in $\mathrm{A}^{0} \quad N_{C}$ is the coordination number and $\lambda$ is the correction factor of the bond ionicity. For group IV-IV, $\lambda=0$, for III-V $\lambda=1$ and $\lambda=2$ for II-VI. In terms of individual bond properties, Grima Gallardo ${ }^{22}$ proposed a modification of Cohen equation(9) and estimated bulk modulus of I-III- $\mathrm{V}_{2}$ chalcopyrite compounds in terms of bond length and the electronic susceptibilities of different I-III and III-V bonds in I-III- $\mathrm{V}_{2}$ chalcopyrite compounds as:

$$
B=(1971-200 \lambda) d^{-3.5}-\left|\chi_{A C}-\chi_{B C}\right|^{2.5}
$$

where $\lambda=0.72, d=\frac{\left(d_{A C}+d_{B C}\right)}{2}$ and $\chi_{A C}$ and $\chi_{B C}$ are electronic susceptibilities of the bonds. Kumar et $a l^{6}$ have studied the bulk modulus of IV, III-V, II-VI, II-IV-V $\mathrm{V}_{2}$ and I-III-VI ${ }_{2}$ semiconductors and proposed an empirical relation for the bulk modulus in terms of Plasmon energy. According to them, the bulk modulus of these semiconductors may be expressed as:

$$
B=P\left(\hbar \omega_{p}\right)^{2.333}\left(B \text { in } \mathrm{GPa}, \hbar \omega_{p} \text { in } \mathrm{eV}\right)
$$

where $P$ constant and its numerical values are 0.109 and 0.125 for I-III-VI $I_{2}$ and II-IV-V $\mathrm{V}_{2}$ respectively. Kumar et al ${ }^{6}$ also modified the relation proposed by Plendl et al ${ }^{15}$ between bulk modulus and microhardness as:

$$
B=k H+\gamma
$$

where $k$ and $\gamma$ are constants. The numerical values of $k$ and $\gamma$ are 16.88 and 19.52 for I-III$\mathrm{VI}_{2}$.Neumann ${ }^{16}$ has proposed the bulk modulus -microhardness relationship in II-IV-V $\mathrm{V}_{2}$ chalcopyrite as

$$
B=\frac{C V_{0}^{-K} H}{T_{m}}
$$

where $C$ and ${ }_{K}$ are adjustable parameters. Considering the ionic charge theory of solids Verma and Bhardwaj ${ }^{12}$ has estimated bulk modulus of ternary chalcopyrite's from lattice thermal conductivity by the following relation:

$$
B=S K^{S+1}
$$

where $S$ is a constant, the numerical value of $S$ is 0.375 and 0.235 for I-III-VI $I_{2}$ and II-IV-V 2 respectively. Reddy et al ${ }^{8,9}$ have correlated bulk modulus with energy gap $\left(E_{g}\right)$ for elemental, II-VI, III$\mathrm{V}$, II-IV-V $\mathrm{V}_{2}$ and I-III-VI $\mathrm{VI}_{2}$ semiconductors respectively. The empirical formula proposed by them may be expressed as: 
$B=13.89 E_{g}+46.90$

(For Elemental, III-V, II-VI and I-III-VI 2 group semiconductors).Later on they have proposed a relation between polarizability and bulk modulus of the above compounds and polarizability has been estimated. Recently Author et $a l^{4}$ have evaluated bulk modulus from Plasmon energy by proposing a linear relation between them and from estimated value of bulk modulus polarizability has been evaluated. The empirical relation between bulk modulus and Plasmon energy proposed by them as:

$$
B=\alpha\left(\hbar \omega_{P}\right)-\beta
$$

where $\alpha$ and $\beta$ are constants and their numerical values are as $\alpha=10 \mathrm{GPa} / \mathrm{eV}$ and $\beta=85$ and $75 \mathrm{~Pa}$ for both ternary chalcopyrite's. Recently Al-Douri et al ${ }^{23-25}$ has studied the bulk modulus of IV, III-V and IIVI semiconductors and proposed an empirical relation for the bulk modulus in terms of the transition pressure, charge density, and lattice constants as:

$$
B=[99-(\lambda+79)]\left(10 P_{t}\right)^{\frac{1}{3}}
$$

where $P_{t}$ is the transition pressure in GPa from $\mathrm{ZB}$ to $\beta-S n$ and $\lambda$ is a parameter appropriate for the group IV $(\lambda=1)$, III-V $(\lambda=5)$ and II-VI $(\lambda=8)$ for semiconductors:

$$
B=[2400-1000 \lambda]\left(S_{C}+1\right)
$$

where, $S_{C}$ is the cation part of the charge density and $\lambda$ is the parameter separating the weak ionic elements from the strong ones, $\lambda=0$ for group IV, $\lambda=1$ for group III-V and $\lambda=1.5$ for II-VI semiconductors.

$$
B=\left[3000-\lambda 1001\left(\frac{a}{2}\right)^{-3.5}\right]
$$

where, $a$ is the lattice constant in $\mathrm{A}^{0}$ and $\lambda$ is an empirical parameter which accounts for the effect of ionicity; $\lambda=0,1,2$ for group IV, III-V and II-VI semiconductors respectively. We studied all the above relations proposed by earlier researchers ${ }^{1-35}$ for the estimation of bulk modulus of ternary chalcopyrite structure solids and motivated to explore new alternative empirical relation to estimate the bulk modulus of the solids. Most of the relations require commercial computer code and high-speed computer with more memory for running the program of these relations for finding the result. In this research work, we had presented an alternative method for the estimation of bulk modulus of ternary chalcopyrite structure solids using a scientific calculator and Excel software. This is quite easy and economical for finding the trends in physical and chemical properties in solid state material science research.

The above-studied relations reveal that bulk modulus of any material depends on the volume of its constituent atoms, bond length, charge density, Plasmon energy, transition pressure, the lattice constant, lattice thermal conductivity and microhardness etc. The volume of the ternary chalcopyrites is related to its specific structure. The structure of ternary chalcopyrites is body center tetragonal (bct). The deformation of the body center tetragonal structure is correlated with three lattice parameter a, $\mathrm{c}$ and $\mathrm{u}$ .These parameters are optimized through minimization of the total energy and accordingly bulk modulus is estimated. The measurement of stiffness of the crystal is related to deformation produced in the crystal. The anisotropic strain of tetragonal structure is reflected by tetragonal deformation (2-c/a). This will also lead to a change in bond length $\left(\mathrm{R}_{\mathrm{AC}} \neq \mathrm{R}_{\mathrm{BC}}\right)$ which reveals anion displacement. This whole scenario will change the bond length and bond strength of the compounds. In order to take into account all these and to define the ground state property of ternary chalcopyrites, we have used two parameters, one parameter is the electronegativity value which is the tendency of an atom to attract electrons to itself during the formation of bonds, another parameter is the principal quantum number, the distance of the valence electron from the nucleus. The longer the distance between the valence electrons and nuclei, means the 
larger the principal quantum number, would show larger electronic polarizability and hence higher bulk modulus. Therefore, electronegativity and principal quantum number, both are assumed to be correlated with the nature of chemical bonding and predicting elastic property like bulk modulus of ternary chalcopyrite structure solids.

For ternary chalcopyrite of I-III-VI $I_{2}$ and II-IV-V $\mathrm{V}_{2}$ type, the graph has been plotted between bulk modulus $(B)$ and the ratio of average principal quantum number of atoms constituting compounds and electronegativity of atoms given in Fig 1. In the plotted figure 1.the values of correlation coefficient $\left(\mathrm{R}^{2}\right)$ are shown. In this case, least square fitting method has been used.

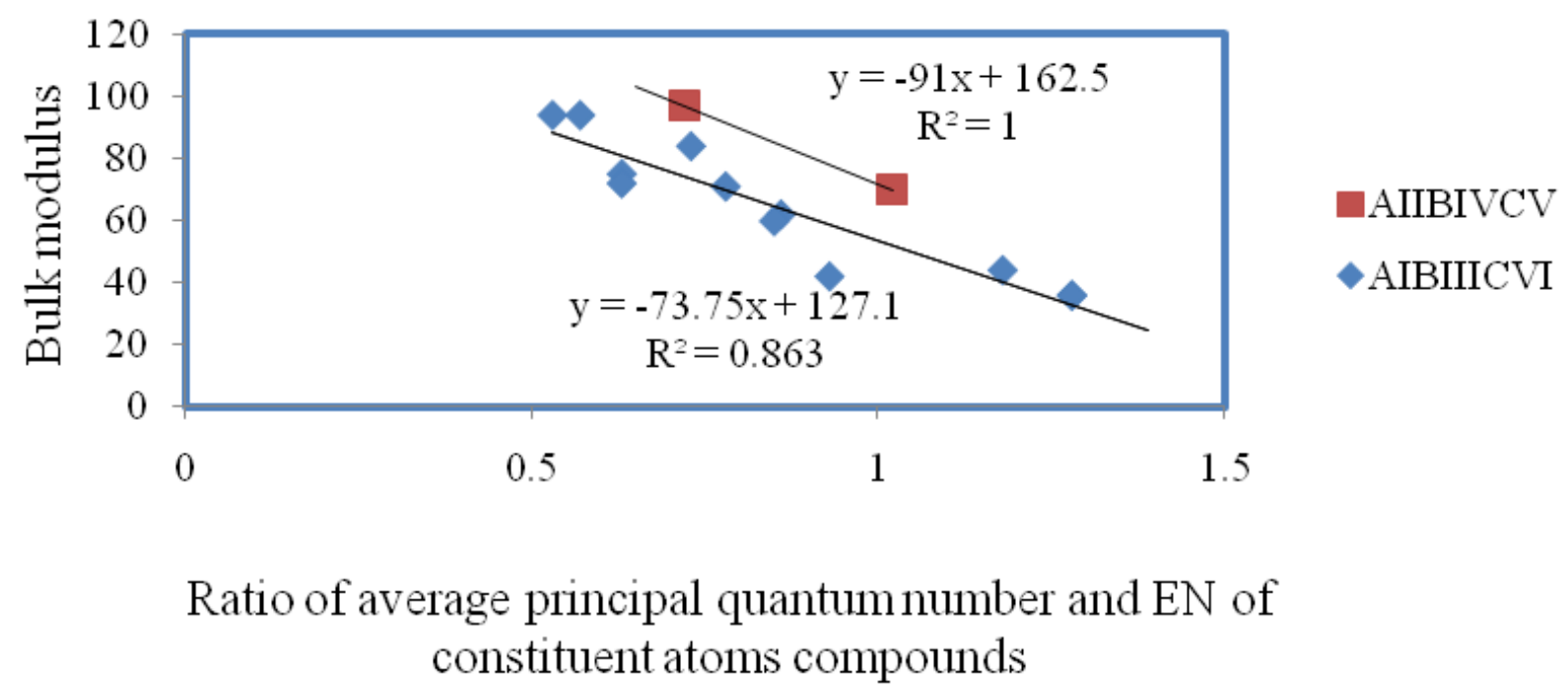

Fig.-1: Plot of bulk modulus Vs ratio of average principal quantum number and EN of constituent atoms of compounds

From the Fig.-1 it is found that bulk modulus $(B)$ and the ratio of average principal quantum number of atoms constituting compounds and electronegativity of atoms are exponentially related, and the $\mathrm{R}^{2}$ value is 0.86 and 1respectively.Therefore the effectiveness of least square fitting is reliable and the normal mathematical expression between bulk modulus $(B)$ and the ratio of average principal quantum number of atoms constituting compounds and electronegativity of atoms is given in equation (22). For ternary chalcopyrite structure solids, the bulk modulus is assumed to be correlated to the contribution of three atoms A, B and C. Considering electronegativity value of two cations A, B and electronegativity value of two anions $\mathrm{C}$ the bulk modulus of ternary chalcopyrite semiconductors can be assumed as $\left(\frac{\eta_{a v}}{\left(\chi_{A} \chi_{B}\right)^{\frac{1}{4}} \chi^{\frac{1}{2}}{ }_{C}}\right)$ where $\eta_{a v}$ is the average principal quantum number of the four constituent atoms in $\mathrm{ABC}_{2,} \eta_{a v}=\left(\eta_{A}+\eta_{B}+2 \eta_{C}\right)^{1 / 4} / 4$. The bulk modulus of ternary chalcopyrite semiconductors can be expressed as:

$B=\alpha\left[\frac{\eta_{a v}}{\left(\chi_{A} \chi_{B}\right)^{\frac{1}{4}} \chi^{\frac{1}{2}} c}\right]+\beta$

where $\alpha$ and $\beta$ have values $-73.75,127.1$ for $\mathrm{I}-\mathrm{III}_{-}-\mathrm{VI}_{2}$ and $-91,162.56$ for $\mathrm{II}-\mathrm{IV}-\mathrm{V}_{2}$ for ternary chalcopyrite structure solids. 
RASĀYAN J. Chem.

Vol. 10 | No. 3 |751 - 758 | July - September | 2017

Table-1: Bulk modulus (GPa) of ternary chalcopyrite $\left(\mathrm{A}^{\mathrm{I}} \mathrm{B}^{\mathrm{III}} \mathrm{C}_{2}{ }^{\mathrm{VI}} \& \mathrm{~A}^{\mathrm{II}} \mathrm{B}^{\mathrm{IV}} \mathrm{C}_{2}{ }^{\mathrm{V}}\right)$ structure solids

\begin{tabular}{|c|c|c|c|c|c|c|c|c|}
\hline Compounds & \multicolumn{8}{|c|}{$\eta_{a v} /\left[\left(\chi_{A} \chi_{B}\right)^{1 / 4} \chi_{C}^{1 / 2}\right]$} \\
\hline $\mathrm{A}^{\mathrm{I}} \mathrm{B}^{\mathrm{IIII}} \mathrm{C}_{2}{ }^{\mathrm{VI}}$ & & & & Eq.(22) & $\begin{array}{r}\text { Ref. } \\
{[6]}\end{array}$ & $\begin{array}{l}\text { Ref. } \\
{[16]}\end{array}$ & $\begin{array}{l}\text { Ref. } \\
{[29]}\end{array}$ & $\begin{array}{l}\text { Ref. } \\
{[30]}\end{array}$ \\
\hline 1 & 2 & & 3 & 4 & 5 & 6 & 7 & 8 \\
\hline $\mathrm{CuAlS}_{2}$ & 0.5 & & $94^{\mathrm{b}}, 99^{\mathrm{c}}$ & 88.01 & 84.02 & 96.8 & 96 & 85.3 \\
\hline $\mathrm{CuAlSe}_{2}$ & 0.7 & & $84^{\mathrm{c}}$ & 73.26 & 69.06 & 77.3 & 80 & 72.9 \\
\hline $\mathrm{CuAlTe}_{2}$ & 1.1 & & & 45.23 & 54.68 & 57.2 & 64 & 53.5 \\
\hline $\mathrm{CuGaS}_{2}$ & 0.5 & & $94^{\mathrm{b}}, 96 \mathrm{~d}, 97^{\mathrm{c}}$ & 85.06 & 82.32 & 95.8 & 94 & 98 \\
\hline $\mathrm{CuGaSe}_{2}$ & 0.7 & & $71^{\mathrm{f}}, 94^{\mathrm{c}}$ & 69.57 & 69.67 & 76.6 & 79 & 69.3 \\
\hline $\mathrm{CuGaTe}_{2}$ & 1.18 & & $44^{\mathrm{g}}$ & 40.07 & 54.24 & 55.4 & 38 & 48.5 \\
\hline $\mathrm{CuInS}_{2}$ & 0.6 & & $75^{\mathrm{h}}$ & 80.63 & 71.73 & 84.4 & 81 & 72.9 \\
\hline $\mathrm{CuInSe}_{2}$ & 0.8 & & $48^{\mathrm{i}}, 53^{\mathrm{j}}, 62^{\mathrm{k}}, 72^{\mathrm{h}}$ & 63.67 & 61.49 & 68.4 & 69 & 64 \\
\hline $\mathrm{CuInTe}_{2}$ & 1.2 & & $36^{\mathrm{j}}, 45.4^{\mathrm{i}}$ & 32.7 & 48.74 & 50.6 & 51 & 45 \\
\hline $\mathrm{AgAlS}_{2}$ & 0.5 & & & 83.58 & 73.19 & 79.9 & 82 & 75.2 \\
\hline $\mathrm{AgAlSe}_{2}$ & 0.8 & & & 68.1 & 56.02 & 65 & 70 & 62.8 \\
\hline $\mathrm{AgAlTe}_{2}$ & 1.2 & & & 37.86 & 56.02 & 48.8 & 54 & 40.4 \\
\hline $\mathrm{AgGaS}_{2}$ & 0.6 & & $60^{\mathrm{d}}, 67^{\mathrm{i}}, 72^{\mathrm{m}}, 90^{\mathrm{n}}$ & 80.63 & 73.19 & 77.6 & 76 & 67.2 \\
\hline $\mathrm{AgGaSe}_{2}$ & 0.8 & & $59.8^{\mathrm{m}}, 65^{\mathrm{n}}$ & 64.41 & 62.15 & 63.8 & 63 & 59.9 \\
\hline $\mathrm{AgGaTe}_{2}$ & 1.2 & & $35.7^{\mathrm{j}}, 71.5^{\mathrm{o}}, 76.6^{\mathrm{m}}$ & 32.7 & 56.02 & 48.6 & 21 & 38.9 \\
\hline $\mathrm{AgInS}_{2}$ & 0.6 & & & 76.21 & 71.52 & 71.6 & 66 & 55 \\
\hline $\mathrm{AgInSe}_{2}$ & 0.9 & & $42^{j}$ & 58.51 & 58.39 & 58.3 & 50 & 48.7 \\
\hline $\mathrm{AgInTe}_{2}$ & 1.3 & & & 24.58 & 43.73 & 44.1 & 29 & 24.5 \\
\hline $\begin{array}{c}\text { Average \% } \\
\text { Deviation }\end{array}$ & & & & 7.5 & 18.8 & 18.08 & 14.8 & 9.23 \\
\hline $\mathrm{A}^{\mathrm{II}} \mathrm{B}^{\mathrm{IV}} \mathrm{C}_{2}{ }^{\mathrm{V}}$ & & & Cal. Eq.(22) & $\begin{array}{r}\text { Ref. } \\
{[6]}\end{array}$ & $\begin{array}{l}\text { Ref. } \\
{[16]}\end{array}$ & $\begin{array}{l}\text { Ref. } \\
{[33]}\end{array}$ & $\begin{array}{l}\text { Ref. } \\
{[34]}\end{array}$ & $\begin{array}{l}\text { Ref. } \\
{[35]}\end{array}$ \\
\hline $\mathrm{ZnSiP}_{2}$ & 0.65 & & 103.35 & 93.13 & 120.3 & 73.2 & 79 & 66.9 \\
\hline $\mathrm{ZnGeP}_{2}$ & 0.71 & & 97.89 & 88.35 & 107.6 & 86 & 73.6 & 71.2 \\
\hline $\mathrm{ZnSnP}_{2}$ & 0.78 & & 91.52 & 75.44 & 84.2 & & 67.5 & 57.7 \\
\hline $\mathrm{ZnSiAs}_{2}$ & 0.86 & & 84.24 & 81.22 & 93.4 & 69.6 & 68 & 53.2 \\
\hline $\mathrm{ZnSeAs}_{2}$ & 0.93 & & 77.87 & 76.68 & 85.7 & 53.8 & 67 & \\
\hline $\mathrm{ZnSnAs}_{2}$ & 1.02 & & 69.68 & 67.43 & 67 & 56.6 & 55.8 & \\
\hline $\mathrm{CdSiP}_{2}$ & 0.72 & $97^{\mathrm{b}}$ & 96.98 & 82.88 & 97 & 71.9 & 48 & 60.9 \\
\hline $\mathrm{CdGeP}_{2}$ & 0.78 & & 91.52 & 75.1 & 86.4 & 67.4 & 47 & 60.4 \\
\hline $\mathrm{CdSnP}_{2}$ & 0.86 & & 84.24 & 67.43 & 67.2 & 55.9 & 52.5 & 50.2 \\
\hline
\end{tabular}


RASĀYAN $J$. Chem.

Vol. 10 | No. 3 |751 - 758 | July - September | 2017

\begin{tabular}{c|c|c|c|c|c|c|c|c}
\hline $\mathrm{CdSiAs}_{2}$ & 0.95 & & 76.05 & 73.19 & & & 54 & \\
\hline $\mathrm{CdGeAs}_{2}$ & 1.02 & $69.7^{\mathrm{p}}$ & 69.68 & 68.28 & 69.7 & 47.7 & 41.3 & \\
\hline $\mathrm{CdSnAs}_{2}$ & 1.11 & & 61.49 & 60.23 & 54.8 & 49.9 & 37.1 & \\
\hline $\begin{array}{c}\text { Average \% } \\
\text { deviation }\end{array}$ & & & 0.02 & 8.29 & 0.00 & 28.72 & 45.63 & 68.60 \\
\hline
\end{tabular}

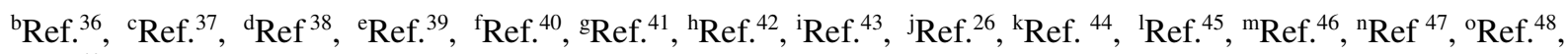
pRef. ${ }^{49}$.

\section{RESULTS AND DISCUSSION}

The bulk modulus of ternary chalcopyrite structure solids is estimated using equation (22). In Table-1, the estimated values are listed along with the results of earlier researchers. ${ }^{6,16,18,26,29,30,36-49}$ It is observed that the calculated values are in better agreement with the experimentally reported values. The results also agree closely with the values reported for these compounds by earlier workers. ${ }^{6-30,36-49}$ The percentage deviation of equation (22) is calculated using the relation lexperimental value-calculated valuel/lexperimental valuel $\times 100$ and percentage deviation are given Table-1. Average percentage deviation of bulk modulus for ternary chalcopyrite's of I-III-VI $I_{2}$ and II-IV-V $\mathrm{V}_{2}$ type has been found to be respectively7.5 and 0.02 for equation (22). For comparison, the average percentage deviation of B for each group of semiconductors is calculated with respect to the values reported by earlier workers. The percentage deviation has been calculated taking the nearest experimental value when more than one experimental values are available for the same ternary chalcopyrite's. It is observed that I-III-VI ${ }_{2}$ compounds show lower bulk modulus than the II-IV-V $\mathrm{V}_{2}$ ternary chalcopyrite structure solids. Within a group, the larger molecular weight, the smaller is the bulk modulus. From the above-estimated values of bulk modulus, it is evident that the hardness of $\mathrm{ZnGeAs}_{2}$ (77.87) exhibits a bulk modulus nearly the same as GaAs (75). It is also observed that bulk modulus amongst the various ternary chalcopyrite structure solids, there is a decreasing trend from sulfide to selenide to telluride in the case of $\mathrm{Cu}-\mathrm{III}-\mathrm{VI}_{2}$ compounds and the same for Ag compounds.

\section{CONCLUSION}

The bulk moduli of ternary chalcopyrites have been empirically estimated using the concept of chemical bonding. In the present study, it is found that electronegativity and average principal quantum number which expresses the nature of bonding in chalcopyrites.It is observed that higher the value of electronegativity of each atom for which the crystal is assumed to be ionic shows less value of bulk modulus, the lower the value electronegativity or more covalency shows higher the value of bulk modulus.

\section{REFERENCES}

1. J. L. Shay and J. H. Wernic Ternary chalcopyrite semiconductors growth, in: Electronic Properties and application, Pergamum Press, Newyork (1975).

2. L. L. Kazmerski, Nuovo Cimento, 20, 1983(2013).

3. S. K. Deab and A. Zunger Ternary Multinary Compounds. Mater. Res. Soc. Conf. Proc, 37, (1987).

4. S. K. Gorai and P. Mahto, Indian J. Phys, 84, 587 (2010).

5. S. K. Gorai and P. Mahto, Indian J. Phys, 86 (4), 273(2012).

6. V. Kumar, A. K. Shrivastava and V. Jha, J. Physics and Chemistry of Solids, 71, 1513(2010).

7. V. Kumar, G. M. Prasad and D. Chandra, Phys. Status. Solidi (b), 170, 77(1992).

8. R. R. Reddy, Y. N .Ahammed, K. Rama Gopal, T. V. R .Rao, Infrared Phys. \& Tech., 3955, (1998).

9. R. R. Reddy A. Y. Nazeer K. Rama Gopal, T. V. R. Rao, P. Abdul Azeem and M. P. Reddy, Optical Materials, 14, 361(1983).

10. A. S. Verma, Phys. Stat. Sol (b), 246, 192(2009).

11. A. S. Verma and D. Sharma, Phys. Scr., 76, 22(2007).

12. A. S. Verma and S. R.Bhardwaj, Phys., Scr, 79, 015302(2009).

13. K. Li and D. Xue, Phys. Status. Solidi B, 2441, 982(2007)

14. D. Xue, S. Zuo and H. Ratajczak, Physica B, 99, 352(2004). 
15. J. N. Plendl, S. S. Mitra and P. Gielisse, J. Phys. Status. Solidi B, 12 367(1965).

16. H. Neumann, Cryst. Res. Technol, 23, 97(1998).

17. M. L. Cohen, Phys. Rev, B,35, 798(1985).

18. P. K. Lam and M. L. Cohen and G. Martinez, Phys. Rev. B, 35, 9198(1987).

19. S. Q. Wang and H. Q. Ye, Physical Review B, 66, 23511(2002).

20. S. Q. Wang and H. Q. Ye, J. Phys: Condensed. Matter, 14, 9579(2002).

21. Eleni Ziambaras and Elisabeth Schroder, Physical Review B, 68, 064112(2003).

22. G. Gallardo, Phys .Status Solidi B, K67, 182(1994).

23. Y. Al- Douri, Material Chemistry and Physics, 78, 625(2003).

24. Y .Al- Douri, H. Abid and H. Aourag, Material Letters, 59, 2032(2005).

25. Y. Al-Douri H. Abid and H. Aourag, Material Chemistry and Physics, 87, 14(2004).

26. D. R. Lide, Hand Book Chemistry and Physics 80 ${ }^{\text {th }}$ ed., CRC Publication, (1999-2000).

27. M. Weber, Hand Book of Optical Materials, CRC Publication (2003).

28. Q. B. Meng, C. Y. Xiao, Z. J. Wu, Ke-an Feng, Z. D. Lin and S.Y. Zhang Solid State Communication, 107, 7, 369(1998).

29. P. G. Gallardo, Phys. Status Solidi B, 182, K67(1984).

30. S. H. Wei, A. Zunger, I.H. Choi P.Y. Yu, Phys. Rev. B, 58, R1710(1998).

31. V. Kumar G. M. Prasad and D. Chandra, Phys. Status Solidi B, 186, K45(1994).

32. A. K .Koh, Phys. Stat. Sol. (b), 209, 25(1998).

33. P. Dues, H. A. Schneider, Cryst.Res. Technol, 20, 867(1985).

34. B. N. Oshcherin, Phys. Status. Solidi A, 51, K175(1979).

35. A. Poplavnoi .S Izv Vyssh, Fizika, 29(8), 5(1986).

36. M. Bettini, W.B. Holzapfel, Solid State Commun, 16, 27(1975).

37. L. Rao, J. C. Chervin, J. P. Itie, A. Polian, M. Gauthier, A. Chevy, Phys. Status. Solidi. B, 211,455(1991).

38. A. Werner, H. D. Hochheimer, A. Jayaraman, Phys. Rev. B, 23, 3836(1981).

39. T. Tinoco, J .P. Itie, A. Polian, A .San, E. Moya Miguel, P. Grima, J. Gonzalez, F. Gonzalez, J. Phys. IV (C9), 151(1994).

40. A. Kraft, G. Kuhn, W. Moller, Z. Anorg.Allg.Chem, 504, 155(1983).

41. A. Kraft, H. Woollstadt, G. Kuhn, W. Moller, Mater. Res. Soc. Symp. Proc, 22,31(1984).

42. T. Tinoco, A. Polian, D. Gomez, Itie. Phys. Status. Solidi B, 198, 433(1996).

43. B. Fernandez, S. M. Wasim, Phys. Status. Solidi. A, 122, 235(1990).

44. R. Fouret, B. Henion, J. Gonzalez, S .M. Wasim, Phys. Rev., 47, 8269(1993).

45. M. H. Grimsditch, G. D. Holah, Phys. Rev, B12, 4377(1975)

46. Y. Mori, K. Taakarabe, S. Iwamoto, S. Minomura, E. Niwa, K. Masumoto, Phys. Status. Solidi., B198, 427(1996).

47. T. Tinoco, A. Polian, J. P. Itie, E. Moya, J. Gonzalez, J. Phys. Chem. Solids, 56,481(1995).

48. Y. Mori, S. I. Iwamoto, K. I. Takarabe, S. Minomura, A .L. Ruoff, Phys. Status. Solidi, B, 221, 469(1999).

49. T. Hailing, G .A. Saunders, W. A. Lampson, R. S. Feigelson, J. Phys. C, 15, 1399(1982).

[RJC-1720/2017] 